\title{
The First Passage Time and the Dividend Value Function for One-Dimensional Diffusion Processes between Two Reflecting Barriers
}

\author{
Chuancun Yin ${ }^{1}$ and Huiqing Wang ${ }^{1,2}$ \\ ${ }^{1}$ School of Mathematical Sciences, Qufu Normal University, Shandong 273165, China \\ ${ }^{2}$ School of Economics and Management, Southeast University, Nanjing 211189, China \\ Correspondence should be addressed to Chuancun Yin, ccyin@mail.qfnu.edu.cn
}

Received 26 July 2012; Accepted 24 September 2012

Academic Editor: Enzo Orsingher

Copyright $\odot 2012$ C. Yin and H. Wang. This is an open access article distributed under the Creative Commons Attribution License, which permits unrestricted use, distribution, and reproduction in any medium, provided the original work is properly cited.

We consider the general one-dimensional time-homogeneous regular diffusion process between two reflecting barriers. An approach based on the Itô formula with corresponding boundary conditions allows us to derive the differential equations with boundary conditions for the Laplace transform of the first passage time and the value function. As examples, the explicit solutions of them for several popular diffusions are obtained. In addition, some applications to risk theory are considered.

\section{Introduction and the Model}

Diffusion processes with one or two barriers appear in many applications in economics, finance, queueing, mathematical biology, and electrical engineering. Among queueing system applications, reflected Ornstein-Uhlenbeck and reflected affine processes have been studied as approximations of queueing systems with reneging or balking [1, 2]. Motivated by Ward and Glynn's one-sided problem, Bo et al. [3] considered a reflected Ornstein-Uhlenbeck process with two-sided barriers. In this paper, we consider the expectations of some random variables involving the first passage time and local times for the general one-dimensional diffusion processes between two reflecting barriers.

Let $X=\left\{X_{t}, t \geq 0\right\}$ be a one-dimensional time-homogeneous reflected diffusion process with barriers $a$ and $b$, which is defined by the following stochastic differential equation:

$$
\begin{gathered}
d X_{t}=\mu\left(X_{t}\right) d t+\sigma\left(X_{t}\right) d B_{t}+d L_{t}-d U_{t}, \\
X_{0}=x \in(a, b),
\end{gathered}
$$


where $B(t)$ is a Brownian motion in $\mathcal{R}, L=\left\{L_{t}, t \geq 0\right\}$ and $U=\left\{U_{t}, t \geq 0\right\}$ are the regulators of point $a$ and $b$, respectively. Further, the processes $L$ and $U$ are uniquely determined by the following properties (see, e.g., [4]):

(1) both $t \rightarrow L_{t}$ and $t \rightarrow U_{t}$ are continuous nondecreasing processes with $L_{0}=U_{0}=0$,

(2) $L$ and $U$ increase only when $X=a$ and $X=b$, respectively, that is, $\int_{0}^{t} I_{\left\{X_{s}=a\right\}} d L_{s}=L_{t}$ and $\int_{0}^{t} I_{\left\{X_{s}=b\right\}} d U_{s}=U_{t}$, for $t \geq 0$.

It is well known that under certain mild regularity conditions on the coefficients $\mu(x)$ and $\sigma(x)$, the $\operatorname{SDE}(1.1)$ has a unique strong solution for each starting point (see, e.g., [5]). The solution $X_{t}$ is a time-homogeneous strong Markov process with infinitesimal generator

$$
\mathscr{A} f(x)=\frac{1}{2} \sigma^{2}(x) f^{\prime \prime}(x)+\mu(x) f^{\prime}(x), \quad x \in(a, b)
$$

acting on functions on $[a, b]$ subject to boundary conditions: $f^{\prime}(a)=f^{\prime}(b)=0$.

Define the first passage time

$$
\tau_{y}=\inf \left\{t \geq 0: X_{t}=y\right\}
$$

where $\tau_{y}=\infty$ if $X_{t}$ never reaches $y$.

For $\lambda>0, \eta>0, \theta>0$, we consider the Laplace transform $\varphi$, and the value functions $\psi, \psi_{1}$, and $\psi_{2}$ on $x \in[a, b]$ :

$$
\begin{gathered}
\varphi(x)=E_{x}\left[e^{-\lambda \tau_{y}}\right] \\
\psi(x)=E_{x}\left[\eta \int_{0}^{\infty} e^{-\lambda t} d U_{t}-\theta \int_{0}^{\infty} e^{-\lambda t} d L_{t}\right], \\
\psi_{1}(x)=E_{x}\left[\int_{0}^{\infty} e^{-\lambda t} d U_{t}\right] \\
\psi_{2}(x)=E_{x}\left[\int_{0}^{\infty} e^{-\lambda t} d L_{t}\right] .
\end{gathered}
$$

The rest of the paper is organized as follows. Section 2 studies the Laplace transform of the first passage time. Section 3 deals with the value function. Some applications in risk theory are considered in Section 4.

\section{Laplace Transform}

Bo et al. [3] consider the Laplace transform $E_{x}\left[e^{-\lambda \tau_{y}}\right]$ for a reflected Ornstein-Uhlenbeck process with two-sided barriers. In this section we consider the Laplace transform of the first passage time for the general reflected diffusion process $X$ defined by (1.1). 
International Journal of Stochastic Analysis

Theorem 2.1. Let $x \in[a, b], \lambda>0$, and assume that $f_{1}(y), f_{2}(y)$ satisfy the following equations, respectively:

$$
\begin{gathered}
\mathcal{A} f_{1}(y)=\lambda f_{1}(y), \quad y \in(a, b), \\
f_{1}^{\prime}(a)=0, \\
\mathcal{A} f_{2}(y)=\lambda f_{2}(y), \quad y \in(a, b), \\
f_{2}^{\prime}(b)=0 .
\end{gathered}
$$

If $f_{1}(y) \neq 0$ for $y \in[x, b]$ and $f_{2}(y) \neq 0$ for $y \in[a, x]$, then

$$
\begin{aligned}
& E_{x}\left[e^{-\lambda \tau_{y}}\right]=\frac{f_{1}(x)}{f_{1}(y)}, \quad y \in[x, b], \\
& E_{x}\left[e^{-\lambda \tau_{y}}\right]=\frac{f_{2}(x)}{f_{2}(y)}, \quad y \in[a, x] .
\end{aligned}
$$

Proof. Applying the Itô formula for semimartingales to $h\left(t, X_{t}\right)=e^{-\lambda t} f\left(X_{t}\right)$ with $f \in$ $C_{b}^{2}([a, b])$ we obtain

$$
\begin{aligned}
h\left(t, X_{t}\right)= & h\left(0, X_{0}\right)+\int_{0}^{t} \frac{\partial h}{\partial s} h\left(s, X_{s}\right) d s+\int_{0}^{t} \frac{\partial h}{\partial x} h\left(s, X_{s}\right) d X_{s} \\
& +\frac{1}{2} \int_{0}^{t} \frac{\partial^{2} h}{\partial x^{2}} h\left(s, X_{s}\right) d\left[X_{s}, X_{s}\right] \\
= & f\left(X_{0}\right)+\int_{0}^{t}-\lambda e^{-\lambda s} f\left(X_{s}\right) d s+\int_{0}^{t} e^{-\lambda s} f^{\prime}\left(X_{s}\right) d X_{s} \\
& +\frac{1}{2} \int_{0}^{t} e^{-\lambda s} f^{\prime \prime}\left(X_{s}\right) \sigma^{2}\left(X_{s}\right) d s \\
= & f(x)+\int_{0}^{t} e^{-\lambda s}(\mathcal{A}-\lambda) f\left(X_{s}\right) d s+\int_{0}^{t} e^{-\lambda s} f^{\prime}\left(X_{s}\right) d B_{s} \\
& +f^{\prime}(a) \int_{0}^{t} e^{-\lambda s} d L_{s}-f^{\prime}(b) \int_{0}^{t} e^{-\lambda s} d U_{s} .
\end{aligned}
$$

Since $\tau_{y}<\infty$ is a stopping time and $x \in[a, b]$, it follows from the optional sampling theorem that

$$
\begin{aligned}
E_{x}\left(e^{-\lambda \tau_{y}} f\left(X_{\tau_{y}}\right)\right)= & f(x)+E_{x}\left(\int_{0}^{\tau_{y}} e^{-\lambda s}(\mathcal{A}-\lambda) f\left(X_{s}\right) d s\right) \\
& +f^{\prime}(a) E_{x}\left(\int_{0}^{\tau_{y}} e^{-\lambda s} d L_{s}\right)-f^{\prime}(b) E_{x}\left(\int_{0}^{\tau_{y}} e^{-\lambda s} d U_{s}\right) .
\end{aligned}
$$


By the definitions of $\tau_{y}, U$, and $L$ we have

$$
E_{x}\left[\int_{0}^{\tau_{y}} e^{-\lambda s} d L_{s}\right]=0, \quad y \in[a, x] ; \quad E_{x}\left[\int_{0}^{\tau_{y}} e^{-\lambda s} d U_{s}\right]=0, \quad y \in[x, b] ; \quad X\left(\tau_{y}\right)=y .
$$

Substituting them into (2.4) one gets

$$
\begin{aligned}
E_{x}\left[e^{-\lambda \tau_{y}} f(y)\right]= & f(x)+E_{x}\left[\int_{0}^{\tau_{y}} e^{-\lambda s}(\mathcal{A}-\lambda) f\left(X_{s}\right) d s\right] \\
& +f^{\prime}(a) E_{x}\left[\int_{0}^{\tau_{y}} e^{-\lambda s} d L_{s}\right], \quad y \in[x, b] . \\
E_{x}\left[e^{-\lambda \tau_{y}} f(y)\right]= & f(x)+E_{x}\left[\int_{0}^{\tau_{y}} e^{-\lambda s}(\mathcal{A}-\lambda) f\left(X_{s}\right) d s\right] \\
& -f^{\prime}(b) E_{x}\left[\int_{0}^{\tau_{y}} e^{-\lambda s} d U_{s}\right], \quad y \in[a, x] .
\end{aligned}
$$

The result follows.

Remark 2.2. Although neither $f_{1}(\cdot)$ nor $f_{2}(\cdot)$ in the Theorem 2.1 is unique, but each of their ratios is unique.

As illustrations of Theorem 2.1, we consider some examples.

Example 2.3. Bessel process: $d X_{t}=\left((d-1) / 2 X_{t}\right) d t+d B_{t}$, where $d>1$ is a real number.

We consider the differential equation $(1 / 2) \psi^{\prime \prime}(x)+((d-1) / 2 x) \psi^{\prime}(x)=\lambda \psi(x), \lambda>0$. It is well known that the increasing and decreasing solutions are, respectively:

$$
\psi_{+}(x)=x^{-v} I_{v}(\sqrt{2 \lambda} x), \quad \psi_{-}(x)=x^{-v} K_{v}(\sqrt{2 \lambda} x),
$$

where $v=(d-2) / 2$ and $I_{v}$ and $K_{v}$ are the usual modified Bessel functions.

Then, we can give $f_{1}(x), f_{2}(x)$ as follows:

$$
f_{1}(x)=C_{1} \psi_{+}(x)+C_{2} \psi_{-}(x), \quad f_{2}(x)=C_{3} \psi_{+}(x)+C_{4} \psi_{-}(x),
$$

where the constants $C_{1}, C_{2}$ and $C_{3}, C_{4}$ can be derived from $f_{1}^{\prime}(a)=0$ and $f_{2}^{\prime}(b)=0$, respectively. We can obtain their ratios, respectively:

$$
\begin{aligned}
& M=\frac{C_{1}}{C_{2}}=-\frac{\sqrt{2 \lambda} a K_{v}^{\prime}(\sqrt{2 \lambda} a)-v K_{v}(\sqrt{2 \lambda} a)}{\sqrt{2 \lambda} a I_{v}^{\prime}(\sqrt{2 \lambda} a)-v I_{v}(\sqrt{2 \lambda} a)}, \\
& N=\frac{C_{3}}{C_{4}}=-\frac{\sqrt{2 \lambda} b K_{v}^{\prime}(\sqrt{2 \lambda} b)-v K_{v}(\sqrt{2 \lambda} b)}{\sqrt{2 \lambda} b I_{v}^{\prime}(\sqrt{2 \lambda} b)-v I_{v}(\sqrt{2 \lambda} b)} .
\end{aligned}
$$


Substituting them into (2.2), we get

$$
\begin{aligned}
& E_{x}\left[e^{-\lambda \tau_{y}}\right]=\frac{M x^{-v} I_{v}(\sqrt{2 \lambda} x)+x^{-v} K_{v}(\sqrt{2 \lambda} x)}{M y^{-v} I_{v}(\sqrt{2 \lambda} y)+y^{-v} K_{v}(\sqrt{2 \lambda} y)}, \quad y \in[x, b], \\
& E_{x}\left[e^{-\lambda \tau_{y}}\right]=\frac{N x^{-v} I_{v}(\sqrt{2 \lambda} x)+x^{-v} K_{v}(\sqrt{2 \lambda} x)}{N y^{-v} I_{v}(\sqrt{2 \lambda} y)+y^{-v} K_{v}(\sqrt{2 \lambda} y)}, \quad y \in[a, x] .
\end{aligned}
$$

Example 2.4. The Ornstein-Uhlenbeck process [6] is as follows:

$$
d X_{t}=v\left(k-X_{t}\right) d t+\sigma d B_{t}, \quad v, \sigma>0, k \in[a, b]
$$

In mathematical finance, the Ornstein-Uhlenbeck process above is known as Vasicek model for the short-term interest rate process [7]. We consider the differential equation $(1 / 2) \sigma^{2} \psi^{\prime \prime}(x)+v(k-x) \psi^{\prime}(x)=\lambda \psi(x)$.

In the case $k=0, \sigma=1$, the two independent solutions to $(1 / 2) \psi^{\prime \prime}(x)-v x \psi^{\prime}(x)=\lambda \psi(x)$ are

$$
\begin{gathered}
\psi_{+}(x)=H_{-\lambda / v}(-\sqrt{v} x)=2^{-\lambda / 2 v} e^{(1 / 2) v x^{2}} D_{-\lambda / v}(-\sqrt{2 v} x), \\
\psi_{-}(x)=H_{-\lambda / v}(\sqrt{v} x)=2^{-\lambda / 2 v} e^{(1 / 2) v x^{2}} D_{\lambda / v}(\sqrt{2 v} x),
\end{gathered}
$$

where $H_{v}(\cdot)$ and $D_{v}(\cdot)$ are, respectively, the Hermite and parabolic cylinder functions [8]. Then, as the way used in Example 2.3, we obtain the ratios of the constants $C_{1}, C_{2}$ and $C_{3}, C_{4}$, respectively:

$$
\begin{gathered}
M=\frac{C_{1}}{C_{2}}=-\frac{v a D_{\lambda / v}(\sqrt{2 v} a)+\sqrt{2 v} D_{\lambda / v}^{\prime}(\sqrt{2 v} a)}{v a D_{-\lambda / v}(\sqrt{2 v} a)-\sqrt{2 v} D_{-\lambda / v}^{\prime}(\sqrt{2 v} a)}, \\
N=\frac{C_{3}}{C_{4}}=-\frac{v b D_{\lambda / v}(\sqrt{2 v} b)+\sqrt{2 v} D_{\lambda / v}^{\prime}(\sqrt{2 v} b)}{v b D_{-\lambda / v}(\sqrt{2 v} b)-\sqrt{2 v} D_{-\lambda / v}^{\prime}(\sqrt{2 v} b)} .
\end{gathered}
$$

Substituting them into (2.2), we get

$$
\begin{aligned}
& E_{x}\left[e^{-\lambda \tau_{y}}\right]=\frac{M e^{(1 / 2) v x^{2}} D_{-\lambda / v}(-\sqrt{2 v} x)+e^{(1 / 2) v x^{2}} D_{\lambda / v}(\sqrt{2 v} x)}{M e^{(1 / 2) v y^{2}} D_{-\lambda / v}(-\sqrt{2 v} y)+e^{(1 / 2) v y^{2}} D_{\lambda / v}(\sqrt{2 v} y)}, \quad y \in[x, b], \\
& E_{x}\left[e^{-\lambda \tau_{y}}\right]=\frac{N e^{(1 / 2) v x^{2}} D_{-\lambda / v}(-\sqrt{2 v} x)+e^{(1 / 2) v x^{2}} D_{\lambda / v}(\sqrt{2 v} x)}{N e^{(1 / 2) v y^{2}} D_{-\lambda / v}(-\sqrt{2 v} y)+e^{(1 / 2) v y^{2}} D_{\lambda / v}(\sqrt{2 v} y)}, \quad y \in[a, x] .
\end{aligned}
$$


For the general $k$ and $\sigma$, the two independent solutions are, respectively

$$
\begin{aligned}
& \psi_{+}(x)=H_{-\lambda / v}\left(-\frac{\sqrt{v}}{\sigma}(x-k)\right)=2^{-\lambda / 2 v} e^{(1 / 2)\left(v / \sigma^{2}\right)(x-k)^{2}} D_{-\lambda / v}\left(-\frac{\sqrt{2 v}}{\sigma}(x-k)\right) \\
& \psi_{-}(x)=H_{-\lambda / v}\left(\frac{\sqrt{v}}{\sigma}(x-k)\right)=2^{-\lambda / 2 v} e^{(1 / 2)\left(v / \sigma^{2}\right)(x-k)^{2}} D_{-\lambda / v}\left(\frac{\sqrt{2 v}}{\sigma}(x-k)\right) .
\end{aligned}
$$

Then, as the way used in Example 2.3, we obtain the ratios of the constants $C_{1}, C_{2}$ and $C_{3}, C_{4}$, respectively

$$
\begin{gathered}
M=\frac{C_{1}}{C_{2}}=-\frac{\sqrt{v}(a-k) D_{-\lambda / v}((\sqrt{2 v} / \sigma)(a-k))+\sqrt{2} \sigma D_{-\lambda / v}^{\prime}((\sqrt{2 v} / \sigma)(a-k))}{\sqrt{v}(a-k) D_{-\lambda / v}(-(\sqrt{2 v} / \sigma)(a-k))-\sqrt{2} \sigma D_{-\lambda / v}^{\prime}(-(\sqrt{2 v} / \sigma)(a-k))}, \\
N=\frac{C_{3}}{C_{4}}=-\frac{\sqrt{v}(b-k) D_{-\lambda / v}((\sqrt{2 v} / \sigma)(b-k))+\sqrt{2} \sigma D_{-\lambda / v}^{\prime}((\sqrt{2 v} / \sigma)(b-k))}{\sqrt{v}(b-k) D_{-\lambda / v}(-(\sqrt{2 v} / \sigma)(b-k))-\sqrt{2} \sigma D_{-\lambda / v}^{\prime}(-(\sqrt{2 v} / \sigma)(b-k))} .
\end{gathered}
$$

Substituting them into (2.2), we get

$$
\begin{aligned}
& E_{x}\left[e^{-\lambda \tau_{y}}\right]=\frac{h(x)\left(M D_{-\lambda / v}(-(\sqrt{2 v} / \sigma)(x-k))+D_{-\lambda / v}((\sqrt{2 v} / \sigma)(x-k))\right)}{h(y)\left(M D_{-\lambda / v}(-(\sqrt{2 v} / \sigma)(y-k))+D_{-\lambda / v}((\sqrt{2 v} / \sigma)(y-k))\right)}, \quad y \in[x, b], \\
& E_{x}\left[e^{-\lambda \tau_{y}}\right]=\frac{h(x)\left(N D_{-\lambda / v}(-(\sqrt{2 v} / \sigma)(x-k))+D_{-\lambda / v}((\sqrt{2 v} / \sigma)(x-k))\right)}{h(y)\left(N D_{-\lambda / v}(-(\sqrt{2 v} / \sigma)(y-k))+D_{-\lambda / v}((\sqrt{2 v} / \sigma)(y-k))\right)}, \quad y \in[a, x],
\end{aligned}
$$

where $h(x)=e^{(1 / 2)\left(v / \sigma^{2}\right)(x-k)^{2}}$.

Remark 2.5. If we take $k=0, \sigma=1, a=0$ and substitute the series forms of $H_{v}(\cdot)$ and $D_{v}(\cdot)$ into the above result, then it is the same as Bo et al. [3].

Example 2.6. The square root process of Cox et al. [9]:

$$
d X_{t}=v\left(k-X_{t}\right) d t+\sigma \sqrt{X_{t}} d B_{t}, \quad v, \sigma>0, k \in[a, b] .
$$

Now consider the differential equation

$$
\frac{1}{2} \sigma^{2} x \psi^{\prime \prime}(x)+(v k-v x) \psi^{\prime}(x)=\lambda \psi(x), \quad \lambda>0 .
$$


If $\left(2 v / \sigma^{2}\right) k$ is not an integer, the two linear independent solutions are

$$
\psi_{+}(x)=M\left(\frac{\lambda}{v}, \frac{2 v}{\sigma^{2}} k, \frac{2 v}{\sigma^{2}} x\right), \quad \psi_{-}(x)=U\left(\frac{\lambda}{v}, \frac{2 v}{\sigma^{2}} k, \frac{2 v}{\sigma^{2}} x\right)
$$

where $M$ and $U$ are the confluent hypergeometric functions of the first and second kinds, respectively. Then, as the way used in Example 2.3, we obtain the ratios of the constants $C_{1}$, $C_{2}$ and $C_{3}, C_{4}$, respectively:

$$
\begin{aligned}
& M_{1}=\frac{C_{1}}{C_{2}}=-\frac{U^{\prime}\left(\lambda / v,\left(2 v / \sigma^{2}\right) k,\left(2 v / \sigma^{2}\right) a\right)}{M^{\prime}\left(\lambda / v,\left(2 v / \sigma^{2}\right) k,\left(2 v / \sigma^{2}\right) a\right)}, \\
& N=\frac{C_{3}}{C_{4}}=-\frac{U^{\prime}\left(\lambda / v,\left(2 v / \sigma^{2}\right) k,\left(2 v / \sigma^{2}\right) b\right)}{M^{\prime}\left(\lambda / v,\left(2 v / \sigma^{2}\right) k,\left(2 v / \sigma^{2}\right) b\right)} .
\end{aligned}
$$

Substituting them into (2.2), we get

$$
\begin{aligned}
& E_{x}\left[e^{-\lambda \tau_{y}}\right]=\frac{M_{1} M\left(\lambda / v,\left(2 v / \sigma^{2}\right) k,\left(2 v / \sigma^{2}\right) x\right)+U\left(\lambda / v,\left(2 v / \sigma^{2}\right) k,\left(2 v / \sigma^{2}\right) x\right)}{M_{1} M\left(\lambda / v,\left(2 v / \sigma^{2}\right) k,\left(2 v / \sigma^{2}\right) y\right)+U\left(\lambda / v,\left(2 v / \sigma^{2}\right) k,\left(2 v / \sigma^{2}\right) y\right)}, \quad y \in[x, b] . \\
& E_{x}\left[e^{-\lambda \tau_{y}}\right]=\frac{N M\left(\lambda / v,\left(2 v / \sigma^{2}\right) k,\left(2 v / \sigma^{2}\right) x\right)+U\left(\lambda / v,\left(2 v / \sigma^{2}\right) k,\left(2 v / \sigma^{2}\right) x\right)}{N M\left(\lambda / v,\left(2 v / \sigma^{2}\right) k,\left(2 v / \sigma^{2}\right) y\right)+U\left(\lambda / v,\left(2 v / \sigma^{2}\right) k,\left(2 v / \sigma^{2}\right) y\right)}, \quad y \in[a, x] .
\end{aligned}
$$

Example 2.7. The Gompertz Brownian motion process [10] is as follows:

$$
d X_{t}=v X(t)\left(\ln (k)-\ln \left(X_{t}\right)\right) d t+\sigma X_{t} d B_{t}, \quad v, \sigma>0, k \in[a, b]
$$

Now consider the differential equation

$$
\frac{1}{2} \sigma^{2} x^{2} \psi^{\prime \prime}(x)+v x(\ln k-\ln x) \psi^{\prime}(x)=\lambda \psi(x), \quad \lambda>0 .
$$

The increasing and decreasing solutions are, respectively:

$$
\begin{aligned}
& \psi_{+}(x)=M\left(\frac{\lambda}{2 v}, \frac{1}{2}, \frac{v}{\sigma^{2}}\left(\ln \frac{x}{k}+\frac{\sigma^{2}}{2 v}\right)^{2}\right), \\
& \psi_{-}(x)=U\left(\frac{\lambda}{2 v}, \frac{1}{2}, \frac{v}{\sigma^{2}}\left(\ln \frac{x}{k}+\frac{\sigma^{2}}{2 v}\right)^{2}\right),
\end{aligned}
$$


where $M$ and $U$, as in Example 2.6, are the first and second Kummer's functions, respectively. Then, as the way used in Example 2.3, we obtain the ratios of the constants $C_{1}, C_{2}$ and $C_{3}, C_{4}$, respectively:

$$
\begin{gathered}
M_{1}=\frac{C_{1}}{C_{2}}=-\frac{U^{\prime}\left(\lambda / 2 v, 1 / 2,\left(v / \sigma^{2}\right)\left(\ln (a / k)+\sigma^{2} / 2 v\right)^{2}\right)}{M^{\prime}\left(\lambda / 2 v, 1 / 2,\left(v / \sigma^{2}\right)\left(\ln (a / k)+\sigma^{2} / 2 v\right)^{2}\right)}, \\
N=\frac{C_{3}}{C_{4}}=-\frac{U^{\prime}\left(\lambda / 2 v, 1 / 2,\left(v / \sigma^{2}\right)\left(\ln (b / k)+\sigma^{2} / 2 v\right)^{2}\right)}{M^{\prime}\left(\lambda / 2 v, 1 / 2,\left(v / \sigma^{2}\right)\left(\ln (b / k)+\sigma^{2} / 2 v\right)^{2}\right)} .
\end{gathered}
$$

Substituting them into (2.2), we get

$$
\begin{aligned}
& E_{x}\left[e^{-\lambda \tau_{y}}\right]=\frac{M_{1} M\left(\lambda / 2 v, 1 / 2,\left(v / \sigma^{2}\right)\left(\ln (x / k)+\sigma^{2} / 2 v\right)^{2}\right)+U(\mathbb{A})}{M_{1} M\left(\lambda / 2 v, 1 / 2,\left(v / \sigma^{2}\right)\left(\ln (x / k)+\sigma^{2} / 2 v\right)^{2}\right)+U(\mathbb{A})}, \quad y \in[x, b], \\
& E_{x}\left[e^{-\lambda \tau_{y}}\right]=\frac{N M\left(\lambda / 2 v, 1 / 2,\left(v / \sigma^{2}\right)\left(\ln (x / k)+\sigma^{2} / 2 v\right)^{2}\right)+U(\mathbb{A})}{N M\left(\lambda / 2 v, 1 / 2,\left(v / \sigma^{2}\right)\left(\ln (x / k)+\sigma^{2} / 2 v\right)^{2}\right)+U(\mathbb{A})}, \quad y \in[a, x],
\end{aligned}
$$

where $(\mathbb{A})$ denotes $\left(\lambda / 2 v, 1 / 2,\left(v / \sigma^{2}\right)\left(\ln (x / k)+\sigma^{2} / 2 v\right)^{2}\right)$.

Remark 2.8. For a certain choice of parameters for $a$ and $b$ in Examples 2.3-2.7, we get the Laplace transform of the first passage time of one-dimensional diffusion with one-sided barrier. For example, letting $a \rightarrow-\infty$ or $b \rightarrow+\infty$ in Example 2.4, one gets the Laplace transform of the first passage time of the Ornstein-Uhlenbeck process with one-sided barrier; see Nobile et al. [11], Ricciardi and Sato [12], Alili et al. [13], and Ditlevsen [6].

\section{The Value Function}

In this section we study the value functions (1.5)-(1.7). Using Itô's formula, we derive differential equation with boundary conditions for $\psi$.

Theorem 3.1. The function $\psi$ defined by (1.5) satisfies the differential equation

$$
\frac{1}{2} \sigma^{2}(x) \psi^{\prime \prime}(x)+\mu(x) \psi^{\prime}(x)=\lambda \psi(x), \quad a<x<b,
$$

with the boundary conditions $\psi^{\prime}(a)=\theta, \psi^{\prime}(b)=\eta$. 
International Journal of Stochastic Analysis

Proof. Applying the Itô's formula for semimartingales to $h\left(t, X_{t}\right)=e^{-\lambda t} \psi\left(X_{t}\right)$ with $\psi \in$ $C_{b}^{2}([a, b])$ we obtain

$$
\begin{aligned}
h\left(t, X_{t}\right)= & h\left(0, X_{0}\right)+\int_{0}^{t} \frac{\partial h}{\partial s} h\left(s, X_{s}\right) d s+\int_{0}^{t} \frac{\partial h}{\partial x} h\left(s, X_{s}\right) d X_{s} \\
& +\frac{1}{2} \int_{0}^{t} \frac{\partial^{2} h}{\partial x^{2}} h\left(s, X_{s}\right) d\left[X_{s}, X_{s}\right] \\
= & \psi\left(X_{0}\right)+\int_{0}^{t}-\lambda e^{-\lambda s} \psi\left(X_{s}\right) d s+\int_{0}^{t} e^{-\lambda s} \psi^{\prime}\left(X_{s}\right) d X_{s} \\
& +\frac{1}{2} \int_{0}^{t} e^{-\lambda s} \psi^{\prime \prime}\left(X_{s}\right) \sigma^{2}\left(X_{s}\right) d s \\
= & \psi(x)+\int_{0}^{t} e^{-\lambda s}(\mathcal{A}-\lambda) \psi\left(X_{s}\right) d s+\int_{0}^{t} e^{-\lambda s} \psi^{\prime}\left(X_{s}\right) d B_{s} \\
& +\psi^{\prime}(a) \int_{0}^{t} e^{-\lambda s} d L_{s}-\psi^{\prime}(b) \int_{0}^{t} e^{-\lambda s} d U_{s},
\end{aligned}
$$

where we have used that $\psi^{\prime}\left(X_{t}\right) d L_{t}=\psi^{\prime}(a) d L_{t}$ and $\psi^{\prime}\left(X_{t}\right) d U_{t}=\psi^{\prime}(b) d U_{t}$. From (3.2) we have

$$
\begin{aligned}
E_{x}\left(\mathrm{e}^{-\lambda t} \psi\left(X_{t}\right)\right)-\psi(x)= & E_{x} \int_{0}^{t} e^{-\lambda s}(\mathcal{A}-\lambda) \psi\left(X_{s}\right) d s \\
& +E_{x}\left(\psi^{\prime}(a) \int_{0}^{t} e^{-\lambda s} d L_{s}-\psi^{\prime}(b) \int_{0}^{t} e^{-\lambda s} d U_{s}\right)
\end{aligned}
$$

Let $f$ be a solution of

$$
\begin{gathered}
\mathscr{A} f(x)=\lambda f(x), \quad x \in(a, b), \\
f^{\prime}(a)=0 \\
f^{\prime}(b)=1 .
\end{gathered}
$$

In place of (3.3), we have

$$
E_{x}\left(\mathrm{e}^{-\lambda t} f\left(X_{t}\right)\right)-f(x)=-\int_{0}^{t} e^{-\lambda s} d U_{s}
$$

Letting $t \rightarrow \infty$, we get

$$
\int_{0}^{\infty} e^{-\lambda s} d U_{s}=f(x)<\infty
$$


Likewise

$$
\int_{0}^{\infty} e^{-\lambda s} d L_{s}<\infty
$$

Letting $t \rightarrow \infty$ in (3.3) and noting (3.6) and (3.7), we get the desired result.

Corollary 3.2. The function $\psi_{1}(x)=E_{x}\left[\int_{0}^{\infty} e^{-\lambda t} d U_{t}\right]$ is solution to the differential equation

$$
\frac{1}{2} \sigma^{2}(x) \psi_{1}^{\prime \prime}(x)+\mu(x) \psi_{1}^{\prime}(x)=\lambda \psi_{1}(x), \quad a<x<b,
$$

with the boundary conditions $\psi_{1}^{\prime}(a)=0, \psi_{1}^{\prime}(b)=1$.

Corollary 3.3. The function $\psi_{2}(x)=E_{x}\left[\int_{0}^{\infty} e^{-\lambda t} d L_{t}\right]$ is solution to the differential equation

$$
\frac{1}{2} \sigma^{2}(x) \psi_{2}^{\prime \prime}(x)+\mu(x) \psi_{2}^{\prime}(x)=\lambda \psi_{2}(x), \quad a<x<b,
$$

with the boundary conditions $\psi_{2}^{\prime}(a)=-1, \psi_{2}^{\prime}(b)=0$.

For diffusions in Examples 2.3-2.7 we can obtain the explicit expressions for $\psi, \psi_{1}$, and $\psi_{2}$. Now we consider the Ornstein-Uhlenbeck process only.

Example 3.4. The Ornstein-Uhlenbeck process is as follows:

$$
d X_{t}=v\left(k-X_{t}\right) d t+\sigma d B_{t}, \quad v, \sigma>0, k \in[a, b] .
$$

From Example 2.4, the two independent solutions of differential equation

$$
\frac{1}{2} \sigma^{2} \psi^{\prime \prime}(x)+v(k-x) \psi^{\prime}(x)=\lambda \psi(x)
$$

are, respectively,

$$
\begin{gathered}
\psi_{+}(x)=H_{-\lambda / v}\left(-\frac{\sqrt{v}}{\sigma}(x-k)\right)=2^{-\lambda / 2 v} e^{(1 / 2)\left(v / \sigma^{2}\right)(x-k)^{2}} D_{-\lambda / v}\left(-\frac{\sqrt{2 v}}{\sigma}(x-k)\right) \\
\psi_{-}(x)=H_{-\lambda / v}\left(\frac{\sqrt{v}}{\sigma}(x-k)\right)=2^{-\lambda / 2 v} e^{(1 / 2)\left(v / \sigma^{2}\right)(x-k)^{2}} D_{-\lambda / v}\left(\frac{\sqrt{2 v}}{\sigma}(x-k)\right) .
\end{gathered}
$$

The general solution of (3.11) is of the form

$$
\psi(x)=C_{1} \psi_{+}(x)+C_{2} \psi_{-}(x),
$$


where the constants $C_{1}$ and $C_{2}$ are determined by the boundary conditions $\psi^{\prime}(a)=\theta, \psi^{\prime}(b)=$ $\eta$. They are

$$
\begin{aligned}
& C_{1}=\frac{\theta \psi_{+}^{\prime}(b)-\eta \psi_{+}^{\prime}(a)}{\psi_{-}^{\prime}(a) \psi_{+}^{\prime}(b)-\psi_{+}^{\prime}(a) \psi_{-}^{\prime}(b)}, \\
& C_{2}=\frac{\eta \psi_{-}^{\prime}(a)-\theta \psi_{-}^{\prime}(b)}{\psi_{-}^{\prime}(a) \psi_{+}^{\prime}(b)-\psi_{+}^{\prime}(a) \psi_{-}^{\prime}(b)} .
\end{aligned}
$$

\section{Applications to Risk Theory}

Let $X_{t}$ denote the surplus of the company. If no dividends were paid, the surplus process follows the stochastic differential equation

$$
d X_{t}=\mu\left(X_{t}\right) d t+\sigma\left(X_{t}\right) d B_{t}, \quad t \geq 0, X_{0}=x,
$$

where $B$ is a Brownian motion and $\mu$ and $\sigma$ are Lipschitz-continuous functions.

The company will pay dividends to its shareholders according to barrier strategy with parameter $b>0$. Whenever the surplus is about to go above the level $b$, the excess will be paid as dividends, and when the surplus is below $b$ nothing is paid out. Let $D(t)$ denote the aggregate dividends by time $t$. Thus the resulting surplus process $Y$ is given by

$$
d Y_{t}=\mu\left(Y_{t}\right) d t+\sigma\left(Y_{t}\right) d B_{t}-d D(t), \quad t \geq 0
$$

Let $T=\inf \left\{t \geq 0: Y_{t}=0\right\}$ be the time of ruin. Note that when $b<\infty$ ruin is certain, that is, $P(T<\infty)=1$. We are interested in the Laplace transform of $T$. This model can be found in Paulsen [14], and some important special cases can be found in Gerber and Shiu [15], Cai et al. [16]. It follows from Theorem 2.1 that, for $0<x<b$ and $\lambda>0, E_{x}\left(\mathrm{e}^{-\lambda T}\right)=h(x)$, where $h$ is the solution of

$$
\begin{gathered}
\mathscr{A} h(x)=\lambda h(x), \quad x \in(0, b), \\
h^{\prime}(b)=0, \quad h(0)=1 .
\end{gathered}
$$

Assume that an insurance company is not allowed to go bankrupt and the beneficiary of the dividends is required to inject capital into the insurance company to keep its risk process stays nonnegative. Under such a dividend policy the controlled risk process with initial reserve $x>0$ satisfies

$$
d \tilde{X}_{t}=\mu\left(\tilde{X}_{t}\right) d t+\sigma\left(\tilde{X}_{t}\right) d B_{t}+d L_{t}-d D_{t}, \quad t \geq 0,
$$


where $L_{t}$ and $D_{t}$ are local times at 0 and $b$, respectively. $L_{t}$ ensures the insurance company will not ruin and $D_{t}$ is the aggregate amount of paid dividends by time $t$. We consider the total expected discounted dividends minus the total expected discounted costs of injected capital:

$$
V(x ; b)=E_{x}\left[\int_{0}^{\infty} e^{-\lambda t} d D_{t}-k \int_{0}^{\infty} e^{-\lambda t} d L_{t}\right],
$$

where $\lambda>0$ is discounted factor and $k$ is the cost per unit injected capital. Avram et al. [17] consider the problem in a Levy processes setting.

According to Theorem 3.1, we obtain the formula $V(x, b)$, as long as we solve the equation

$$
\frac{1}{2} \sigma^{2}(x) V^{\prime \prime}(x)+\mu(x) V^{\prime}(x)=\lambda V(x), \quad 0<x<b,
$$

with the boundary conditions $V^{\prime}(0)=k, V^{\prime}(b)=1$.

For $0<x<b$, let $T_{x}=\inf \left\{t \mid \widetilde{X}_{t}=x\right\}$ be the first time when the surplus reaches the level $x$. It follows from Theorem 2.1 that, for $0<x<b$ and $\lambda>0, E_{x}\left(\mathrm{e}^{-\lambda T_{x}}\right)=g(x)$, where $g$ is the solution of

$$
\begin{gathered}
A g(x)=\lambda g(x), \quad x \in(0, b), \\
g^{\prime}(0)=0, \quad g(b)=1 .
\end{gathered}
$$

We now give two examples.

Example 4.1. In this example we consider the uncontrolled surplus of insurance company satisfying $X_{t}=x+\mu t+\sigma W_{t}$, where $W_{t}$ is Brownian motion. The controlled surplus process $\tilde{X}$ at time $t$ follows the equation

$$
\tilde{X}_{t}=x+\mu t+\sigma W_{t}+L_{t}-D_{t} .
$$

Now we consider the differential equation

$$
\frac{1}{2} \sigma^{2} V^{\prime \prime}(x)+\mu V^{\prime}(x)=\lambda V(x), \quad 0<x<b,
$$

with the boundary conditions $V^{\prime}(0)=k, V^{\prime}(b)=1$. Then

$$
V(x, b)=\frac{k e^{r b}-1}{s\left(e^{r b}-e^{s b}\right)} e^{s x}-\frac{k e^{s b}-1}{r\left(e^{r b}-e^{s b}\right)} e^{r x}, \quad 0<x<b,
$$


where $r$ and $s$ are the positive root and negative root of the equation $\left(\sigma^{2} / 2\right) \xi^{2}+\mu \xi-\lambda=0$, respectively, that is,

$$
r=\frac{-\mu+\sqrt{\mu^{2}+2 \sigma^{2} \lambda}}{\sigma^{2}}, \quad s=\frac{-\mu-\sqrt{\mu^{2}+2 \sigma^{2} \lambda}}{\sigma^{2}} .
$$

For $0<x<b$ and $\lambda>0, E_{x}\left(\mathrm{e}^{-\lambda T_{x}}\right):=g(x)$ is the solution of

$$
\frac{1}{2} \sigma^{2} g^{\prime \prime}(x)+\mu g^{\prime}(x)=\lambda g(x), \quad 0<x<b,
$$

with the boundary conditions $g^{\prime}(0)=0, g(b)=1$. Solving it gives

$$
E_{x}\left(\mathrm{e}^{-\lambda T_{x}}\right)=\frac{r \mathrm{e}^{s x}-s \mathrm{e}^{r x}}{r \mathrm{e}^{s b}-s \mathrm{e}^{r b}}, \quad 0<x<b
$$

Example 4.2. In this example we consider the Ornstein-Uhlenbeck-type model. The company's surplus evolves according to

$$
\widetilde{X_{t}}=x+\int_{0}^{t}\left(\mu+\rho \widetilde{X_{s}}\right) d s+\sigma B_{t}+L_{t}-D_{t}
$$

The model is considered in Cai et al. [16] for the special case where $L_{t}=0$.

The diffusion and drift coefficients are $\sigma(x) \equiv \sigma, \mu(x)=\mu+\rho x$. We consider the differential equation

$$
\frac{1}{2} \sigma^{2} V^{\prime \prime}(x, b)+(\mu+\rho x) V^{\prime}(x, b)=\lambda V(x, b), \quad 0<x<b,
$$

with the boundary conditions $V^{\prime}(0)=k, V^{\prime}(b)=1$. In Cai et al. [16], they pointed out that the solution is given by

$$
V(x, b)=H(t)=C_{1}(-t)^{1-c} e^{t} M(1-a, 2-c ;-t)+C_{2} e^{t} U(c-a, c ;-t)
$$

for certain coefficients $C_{1}$ and $C_{2}$, with $c=1 / 2, a=-\lambda / 2 \rho, t=-\left(1 / \rho \sigma^{2}\right)(\mu+\rho x)^{2}$. Here $M$ and $U$ are called the confluent hypergeometric functions of the first and second kinds, respectively. For more details on confluent hypergeometric functions, see Abramowitz and Stegun [8]. It follows from (3.7) in Cai et al. [16] that

$$
\begin{aligned}
H^{\prime}(t)= & H(t)-C_{1}(1-c)(-t)^{-c} e^{t} M(1-a, 2-c ;-t) \\
& -C_{1} \frac{1-a}{2-c}(-t)^{1-c} e^{t} M(2-a, 3-c ;-t) \\
& +C_{2}(c-a) e^{t} U(c-a+1, c+1 ;-t) .
\end{aligned}
$$


The conditions $C_{1}$ and $C_{2}$ can be determined by $V^{\prime}(0)=k, V^{\prime}(b)=1$, where $V^{\prime}(x)=-(2(\mu+$ $\left.\rho x) / \sigma^{2}\right) H^{\prime}(t)$. Solving it gives

$$
\begin{aligned}
& C_{1}=\frac{\sigma^{2}}{2} \frac{(C /(\mu+\rho x)) e^{(\mu+\rho b)^{2} / \rho \sigma^{2}}-(k D / \mu) e^{\mu^{2} / \rho \sigma^{2}}}{A D-B C} \\
& C_{2}=\frac{\sigma^{2}}{2} \frac{(k B / \mu) e^{\mu^{2} / \rho \sigma^{2}}-(A /(\mu+\rho b)) e^{(\mu+\rho b)^{2} / \rho \sigma^{2}}}{A D-B C}
\end{aligned}
$$

where

$$
\begin{aligned}
A= & \left(\left(\frac{\mu^{2}}{\rho \sigma^{2}}\right)^{1 / 2}-\frac{1}{2}\left(\frac{\mu^{2}}{\rho \sigma^{2}}\right)^{-1 / 2}\right) M\left(1-a, 2-c ; \frac{\mu^{2}}{\rho \sigma^{2}}\right) \\
& -\frac{1-a}{2-c}\left(\frac{\mu^{2}}{\rho \sigma^{2}}\right)^{1 / 2} M\left(2-a, 3-c ; \frac{\mu^{2}}{\rho \sigma^{2}}\right), \\
B= & \left(\left(\frac{(\mu+\rho b)^{2}}{\rho \sigma^{2}}\right)^{1 / 2}-\frac{1}{2}\left(\frac{(\mu+\rho b)^{2}}{\rho \sigma^{2}}\right)^{-1 / 2}\right) M\left(1-a, 2-c ; \frac{(\mu+\rho b)^{2}}{\rho \sigma^{2}}\right) \\
& -\frac{1-a}{2-c}\left(\frac{(\mu+\rho b)^{2}}{\rho \sigma^{2}}\right)^{1 / 2} M\left(2-a, 3-c ; \frac{(\mu+\rho b)^{2}}{\rho \sigma^{2}}\right), \\
C=U\left(c-a, c ; \frac{\mu^{2}}{\rho \sigma^{2}}\right)+(c-a) U\left(c-a+1, c+1 ; \frac{\mu^{2}}{\rho \sigma^{2}}\right), & \left(c-a, c ; \frac{(\mu+\rho b)^{2}}{\rho \sigma^{2}}\right)+(c-a) U\left(c-a+1, c+1 ; \frac{(\mu+\rho b)^{2}}{\rho \sigma^{2}}\right) .
\end{aligned}
$$

\section{Acknowledgments}

The authors thank the reviewer for valuable insights and suggestions that largely contributed to the improvement of the paper. The research was supported by the National Natural Science Foundation of China (no. 11171179) and the Research Fund for the Doctoral Program of Higher Education of China (no. 20093705110002).

\section{References}

[1] A. R. Ward and P. W. Glynn, "A diffusion approximation for a Markovian queue with reneging," Queueing Systems, vol. 43, no. 1-2, pp. 103-128, 2003.

[2] A. R. Ward and P. W. Glynn, "Properties of the reflected Ornstein-Uhlenbeck process," Queueing Systems, vol. 44, no. 2, pp. 109-123, 2003.

[3] L. Bo, L. Zhang, and Y. Wang, "On the first passage times of reflected Ornstein-Uhlenbeck processes with two-sided barriers," Queueing Systems, vol. 54, no. 4, pp. 313-316, 2006.

[4] M. Harrison, Brownian Motion and Stochastic Flow Systems, John Wiley \& Sons, New York, NY, USA, 1986. 
[5] P.-L. Lions and A.-S. Sznitman, "Stochastic differential equations with reflecting boundary conditions," Communications on Pure and Applied Mathematics, vol. 37, no. 4, pp. 511-537, 1984.

[6] S. Ditlevsen, "A result on the first-passage time of an Ornstein-Uhlenbeck process," Statistics $\mathcal{E}$ Probability Letters, vol. 77, no. 18, pp. 1744-1749, 2007.

[7] O. Vasicek, "An equilibrium characterization of the term structure," Journal of Financial Economics, vol. 5, no. 2, pp. 177-188, 1977.

[8] M. Abramowitz and I. A. Stegun, Handbook of Mathematical Functions: With Formulas, Graphs, and Mathematical Tables, U.S. Government Printing Office, Washington, DC, USA, 1972.

[9] J. C. Cox, J. E. Ingersoll Jr., and S. A. Ross, "A theory of the term structure of interest rates," Econometrica, vol. 53, no. 2, pp. 385-407, 1985.

[10] L. Wang and K. Pötzelberger, "Crossing probabilities for diffusion processes with piecewise continuous boundaries," Methodology and Computing in Applied Probability, vol. 9, no. 1, pp. 21-40, 2007.

[11] A. G. Nobile, L. M. Ricciardi, and L. Sacerdote, "A note on first-passage time and some related problems," Journal of Applied Probability, vol. 22, no. 2, pp. 346-359, 1985.

[12] L. M. Ricciardi and S. Sato, "First-passage-time density and moments of the Ornstein-Uhlenbeck process," Journal of Applied Probability, vol. 25, no. 1, pp. 43-57, 1988.

[13] L. Alili, P. Patie, and J. L. Pedersen, "Representations of the first hitting time density of an OrnsteinUhlenbeck process," Stochastic Models, vol. 21, no. 4, pp. 967-980, 2005.

[14] J. Paulsen, "Optimal dividend payouts for diffusions with solvency constraints," Finance and Stochastics, vol. 7, no. 4, pp. 457-473, 2003.

[15] H. U. Gerber and E. S. W. Shiu, “Optimal dividends: analysis with Brownian motion," North American Actuarial Journal, vol. 8, no. 1, pp. 1-20, 2004.

[16] J. Cai, H. U. Gerber, and H. Yang, "Optimal dividends in an Ornstein-Uhlenbeck type model with credit and debit interest," North American Actuarial Journal, vol. 10, no. 2, pp. 94-119, 2006.

[17] F. Avram, Z. Palmowski, and M. R. Pistorius, "On the optimal dividend problem for a spectrally negative Lévy process," The Annals of Applied Probability, vol. 17, no. 1, pp. 156-180, 2007. 


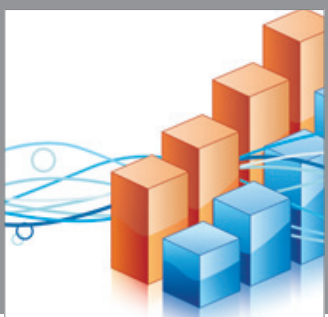

Advances in

Operations Research

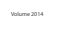

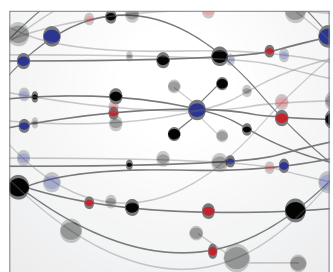

\section{The Scientific} World Journal
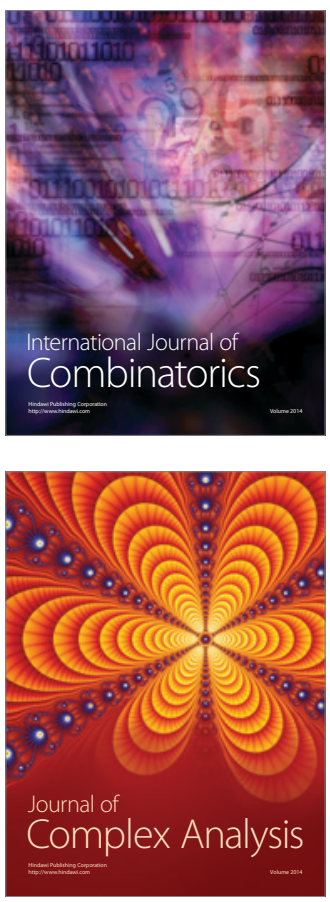

International Journal of

Mathematics and

Mathematical

Sciences
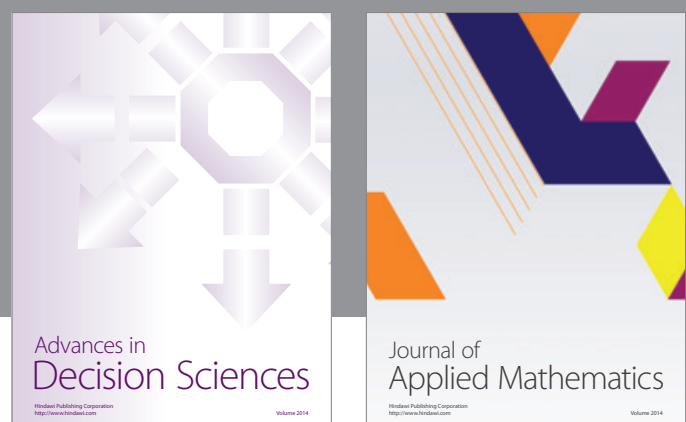

Journal of

Applied Mathematics
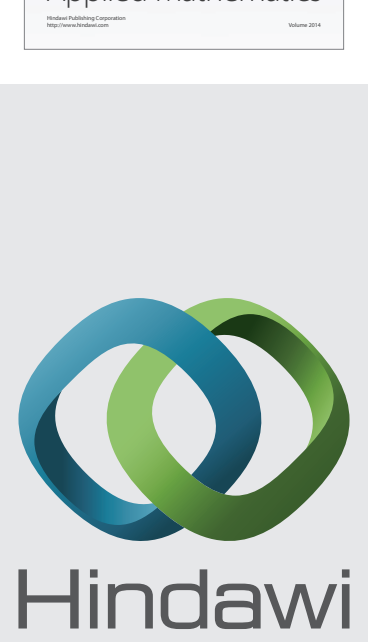

Submit your manuscripts at http://www.hindawi.com
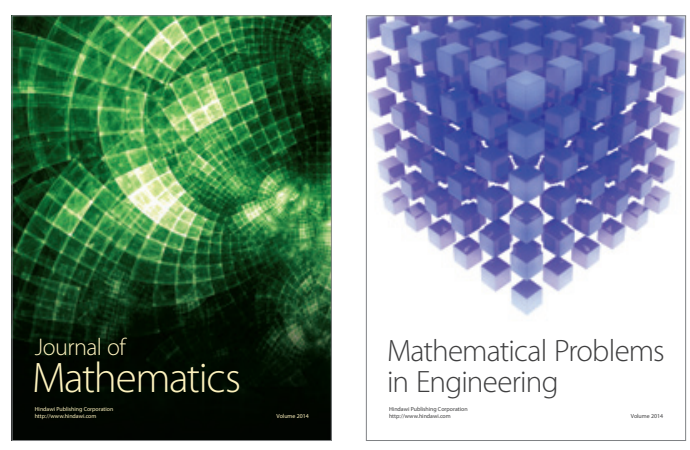

Mathematical Problems in Engineering
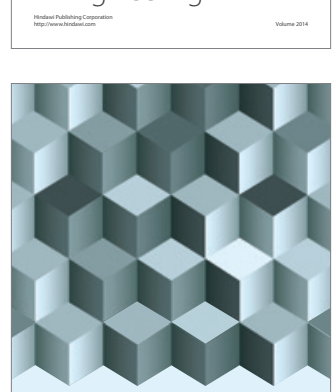

Journal of

Function Spaces
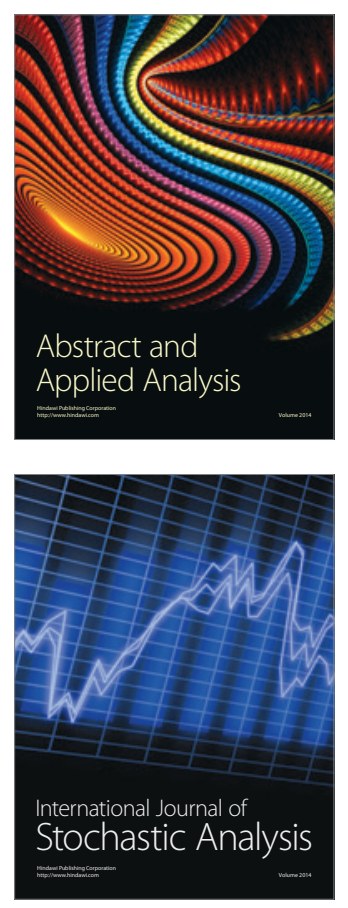

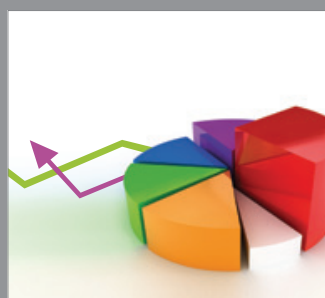

ournal of

Probability and Statistics

Promensencen
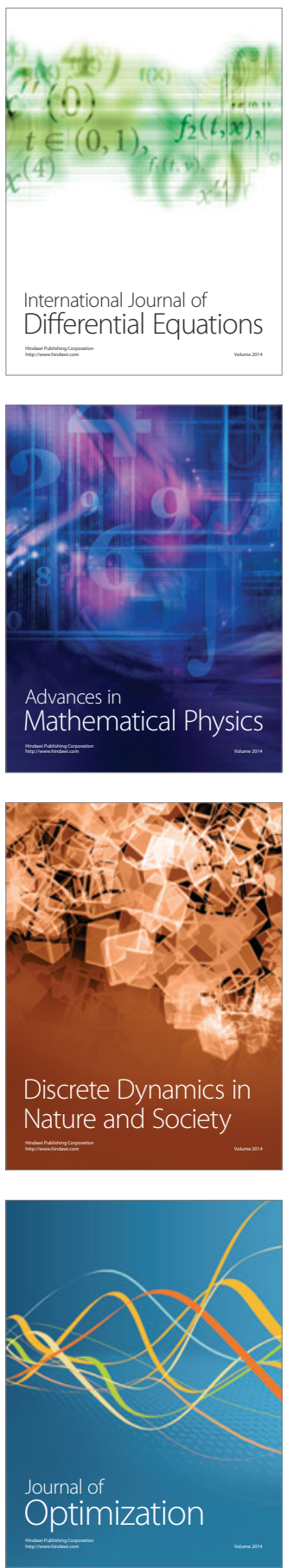